\title{
Research Paper \\ Effect of Pilates Exercises on Motor Performance and Low Back Pain in Elderly Women With Abdominal Obesity
}

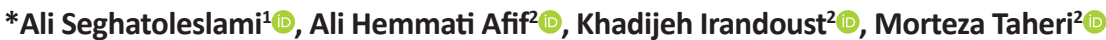

1. Department of Physical Education, Faculty of Physical Education, University of Birjand, Birjand, Iran.

2. Department of Physical Education and Sports Science, Faculty of Social Sciences, Imam Khomeini International University, Qazvin, Iran.

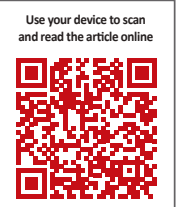

Citation: Seghatoleslami A, Hemmati Afif A, Irandoust Kh, Taheri M. [Effect of Pilates Exercises on Motor Performance and Low Back Pain in Elderly Women With Abdominal Obesity (Persian)]. Iranian Journal of Ageing. 2018; 13(3):396-405. https://doi.org/10.32598/SIJA.13.3.396

https://doi.org/10.32598/SIJA.13.3.396

Keywords:

Pilates exercise, Elderly, Body composition, Low back pain

\section{A B STRACT}

Objectives This research aimed to investigate the impact of Pilates exercise on motor performance indexes and low back pain of inactive middle-aged women.

Methods \& Materials This was a quasi-experimental study with pretest and posttest design performed on 43 inactive females with mean (SD) age of 44.4 (5.1) year with low activity, body fat percentage above $35 \%$, and Waist Hip Ratio (WHR) of over 0.95 . They were assigned into test $(n=22)$ and control $(n=21)$ group. The Pilates or test group did their exercise for 4 months, three times a week while the control group did their daily routines. All body composition variables were measured by body composition analyzer and functional tests were measured by speed and balance tests. Independent and dependent $t$ test were used to analyze the data.

Results The results suggested that dynamic balance and speed improved significantly and those effective factors on motor control such as obesity variables, including Body Mass Index (BMI), WHR, body fat percentage, visceral fat were also improved $(\mathrm{P} \leq 0.05)$.

Conclusion It seems that Pilates exercise may improve motor performance indexes and also a better quality of life due to a mitigating low back pain. Therefore, practicing such an exercise program can be effective in older persons.

\section{Extended Abstract}

\section{Introduction}

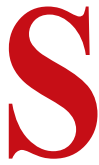

tudies indicate the progressive degeneration of the nervous system and loss of physiological function in elderly people [1]. Since musculoskeletal disorders are one of the most common problems in the elderly, it is important to provide a strategy for its prevention or control. In this regard, low back pain is more common in people with inactive lifestyle [2], increased body mass index, especially in the abdominal region, and muscle weakness in central areas. With the increase in abdominal obesity, the compensatory curve is created in the lumbar curvature which increases pressure on the intervertebral discs [2]. Due to the increase in the curves of the lumbar region, the center of gravity moves forward and causes changes in how to walk, disturbance in postural control and increased risk of falling, and finally reduction in motor function [3], including a reduction

\section{* Corresponding Author:}

Ali Seghatoleslami, PhD

Address: Department of Physical Education, Faculty of Physical Education, University of Birjand, Birjand, Iran.

Tel: +98 (915) 5623745

E-mail: aseghatoleslami@birjand.ac.ir 
Table 1. Results of t test for dynamic balance measurement before and after intervention

\begin{tabular}{|c|c|c|c|c|}
\hline \multirow{2}{*}{ Group } & \multicolumn{2}{|c|}{ Mean $\pm S D$} & \multirow{2}{*}{$\mathbf{t}$} & \multirow{2}{*}{ Sig.* } \\
\hline & Pretest & Posttest & & \\
\hline Test & $8.28 \pm 0.40$ & $7.54 \pm 0.33$ & 6.55 & *0.001 \\
\hline Control & $8.05 \pm 0.41$ & $7.98 \pm 0.29$ & 0.511 & 0.61 \\
\hline
\end{tabular}

$* \mathrm{P}<0.005$.

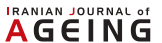

in walking speed and balance that results in reduced functional independence. In this regard, this study aimed to examine the effect of Pilates exercises on motor performance and low back pain in elderly women.

\section{Methods and Materials}

This study is a quasi-experimental study with control group using pretest and posttest conducted on 43 inactive elderly women with a mean (SD) age of 64.4 (5.1) year. The subjects were randomly divided into groups of test $(n=22)$ and control $(n=21)$. The Physical Activity Readiness Questionnaire (PAR-Q) was used to select samples to enter the study [2]. The test group performed Pilates exercises 3 sessions per week for 4 months. The inclusion criteria were as follows: 60 years or older, with non-specific low back pain in the past year, body fat percentage (BFP) higher than 35\%, waist-hip ratio above 0.90 , no problem for performing exercises, no medication use, and no history of lower limb injury during the past year. This study was approved by Ethics Committee of Imam Khomeini International University under code No. 17682. The Keele
STarT Back Screening Tool (SBST) (9-item version) was used to measure low back pain which help physicians identify psychological and physical risk factors for pain [3]. The obesity variables (BFP and visceral fat) were measured by inBody 320 Body Composition Analyzer (InBody Co., Korea) [4]. To measure the speed of the subjects, $10-\mathrm{m}$ sprint test, and to predict the risk of falling, Timed Up and Go test (TUG) were applied. Independent and dependent t test were performed to analyze the collected data.

\section{Result}

The results of this study showed that muscle volume $(\mathrm{P}=0.03)$ and abdominal obesity $(\mathrm{P}=0.001)$ in subjects with physical activity (test group) significantly improved compared to the control group. Physical (Figure 1a) and psychological symptoms of low back pain (Figure 1b) significantly improved after intervention with Pilates exercises $(\mathrm{P}=0.001)$.

The results of dependent $t$ test presented in Table 1 , indicate that the dynamic balance of participants in test

Table 2. Results of t test for walking speed measurement before and after the intervention

\begin{tabular}{|c|c|c|c|c|}
\hline \multirow{2}{*}{ Group } & \multicolumn{2}{|c|}{ Mean士SD } & \multirow{2}{*}{$\mathbf{t}$} & \multirow{2}{*}{ Sig.* } \\
\hline & Pretest & Posttest & & \\
\hline Test & $1.90 \pm 0.03$ & $1.85 \pm 0.04$ & 5.801 & ${ }^{*} 0.001$ \\
\hline Control & $1.91 \pm 0.04$ & $1.92 \pm 0.03$ & 0.054 & 0.958 \\
\hline
\end{tabular}

$* \mathrm{P}<0.005$.

$\stackrel{\text { IRanan ounna oo }}{A}$ GEING

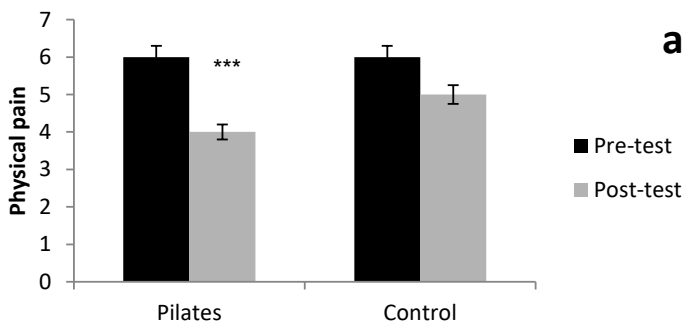

a

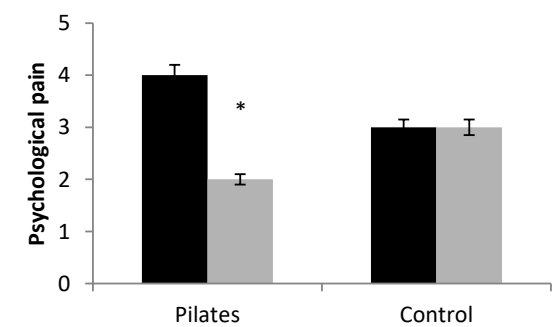

b

Figure 1. Low back pain measurement results 
group significantly improved $(\mathrm{P}=0.001, \mathrm{t}=6.55)$, while there was no significant difference in control group $(\mathrm{t}=0.51, \mathrm{P}=0.616)$. According to the walking speed results in Table 2, there was a significant difference in the posttest results of test group $(\mathrm{P}=0.001, \mathrm{t}=5.801)$, while no significant difference was found in control group $(\mathrm{P}=0.958, \mathrm{t}=0.054)$.

\section{Conclusion}

The positive effects of Pilates exercises on the variables of body composition, back pain, and walking speed will improve the functional independence of the elderly. It was found out that Pilates exercises can reduce the physical and psychological symptoms of low back pain as well as abdominal and visceral obesity, and improve balance and walking speed. Since there was a direct relationship between abdominal obesity and low back pain; one of the possible causes of improving back pain in the Pilates group is the reduction in abdominal obesity. The mechanism of Pilates exercise training focuses on the development of comprehensive power where strength and endurance of the muscles increase; this improvement in the open-chain movement is effective in controlling the movement of the lower and upper limbs which results in improvement of functional factors [6]. Hence, Pilates exercises can improve the motor function of the elderly and cause a functional independency.

\section{Ethical Considerations}

\section{Compliance with ethical guidelines}

This study was referred to the Ethics and Research Council of Imam Khomeini International University for ethical review, and was performed after obtaining permission from the committee (No. 17682) was taken. Before the study began, the research process including the research objectives, how to measure variables, how to do the research program, and the length of the research period was explained to the participants. All participants volunteered to participate in the study, completing the consent.

\section{Funding}

This research did not receive any specific grant from funding agencies in the public, commercial, or not-forprofit sectors.

\section{Conflict of interest}

All authors certify that this manuscript has neither been published in whole nor in part nor being considered for publication elsewhere.

\section{Acknowledgements}

Hereby, we appreciate all the participants who helped us in conducting this research. 


\title{
تأثير يك دوره تمرينات ييلاتس بر شاخصهاى عملكرد حركتى و ميزان كمردرد زنان سالمند
}

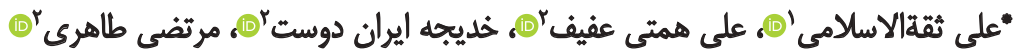

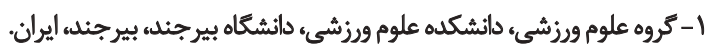

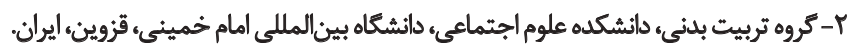

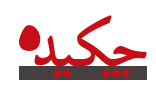

هذأق هدف از انجام مطالعه حاضر، تأثير تمرينات بيلاتس بر شاخصهاى عملكرد حركتى و ميزان كمردرد زنان سالمند بود.

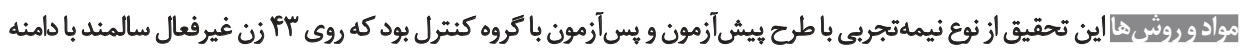

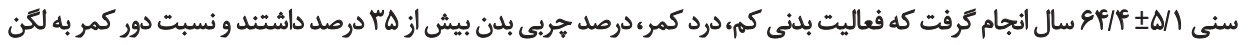

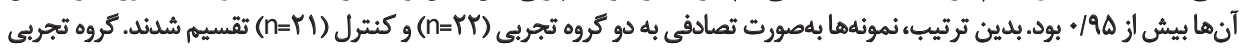

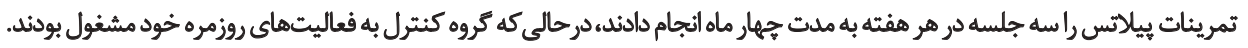

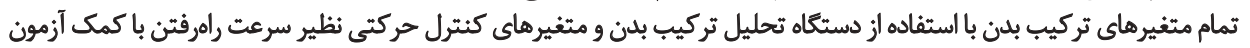

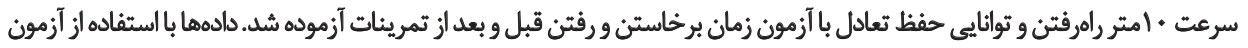

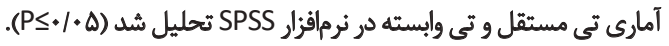

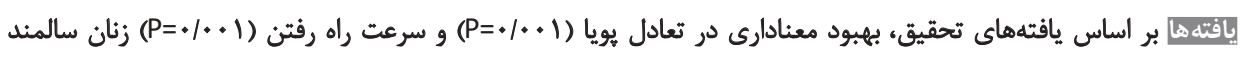

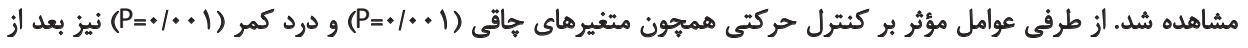

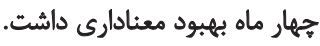

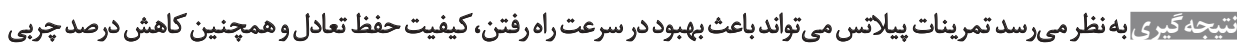

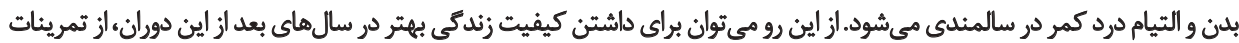

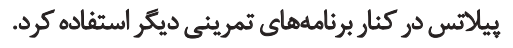

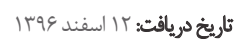

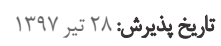

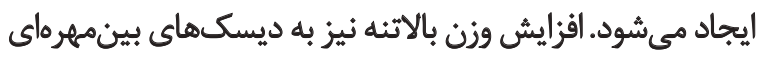

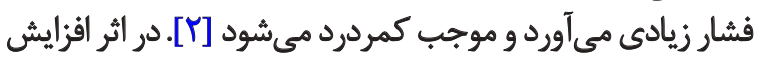

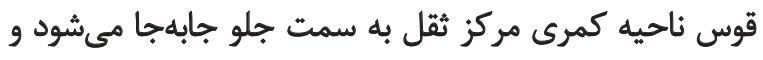

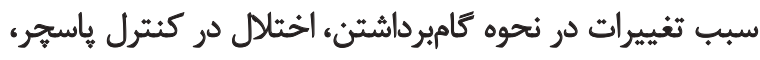

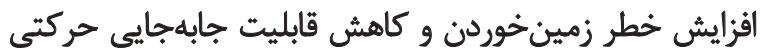

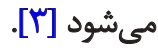

يكى از روشهاى ورزشى كه در سالهاى اخير متخصصان

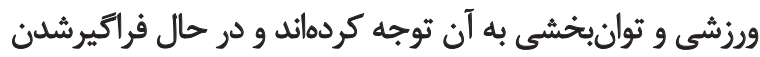

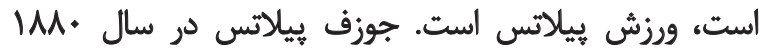

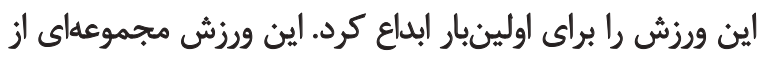

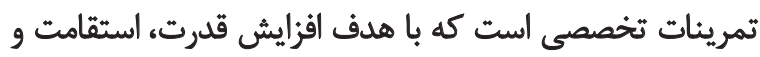

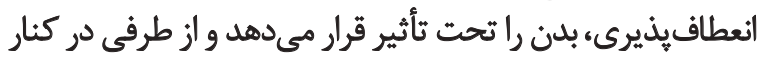

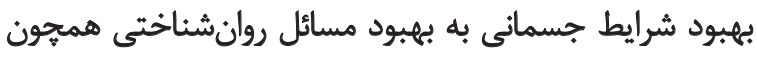

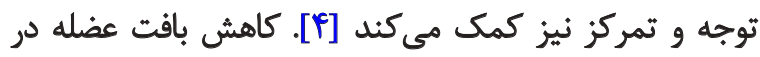

مقدمه امروزه به دليل اقتضائاتى كه در كشور وجود دارد، نسبت

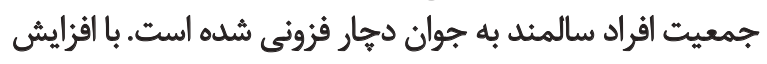

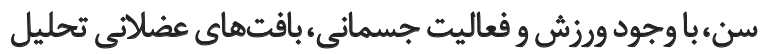

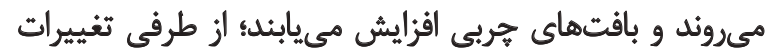

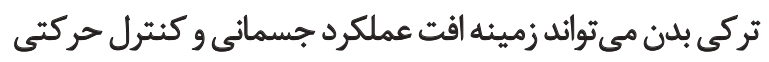

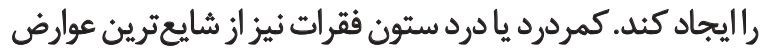

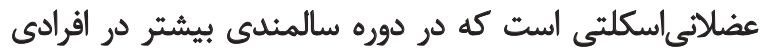

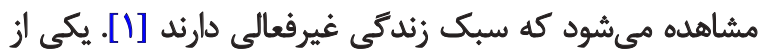

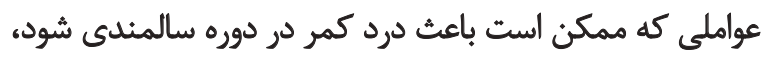

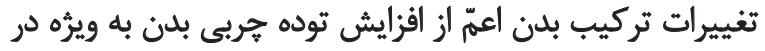

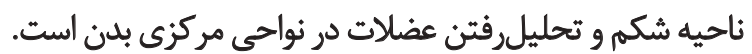
با افزايش جاقى شكمى، قوسى جبرانى در ناحيه انحناى كمرى 
بركماى شامل هفت سؤال بلى و خير است كه براى

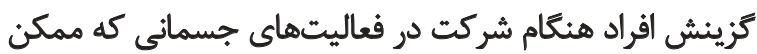

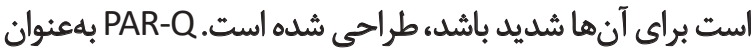

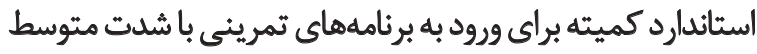

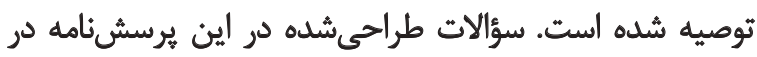

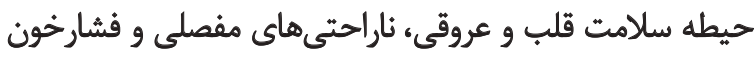

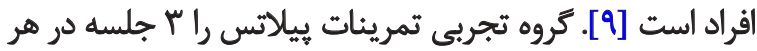

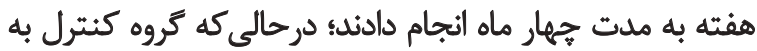

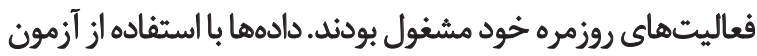
آمارى تى مستقل و تى وابسته در نرم|فزار SPSS تحليل شدندا كاند.

\section{معيار هاي ورو و به مطالع}

تمايل به شركت در تحقيق (امضاى فرم رضايتنامه)، محدوده

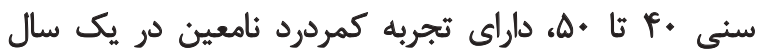

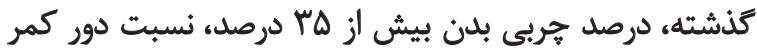

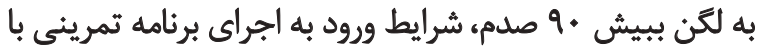

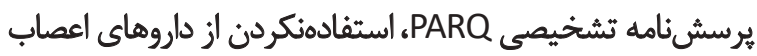

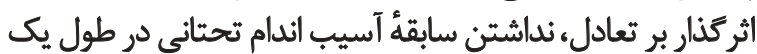

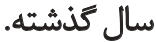

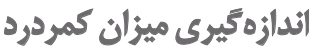

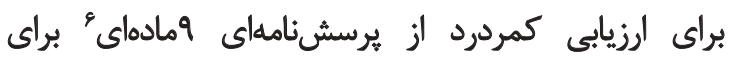

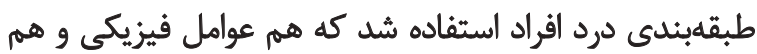

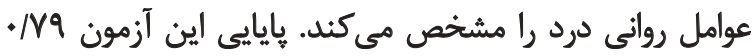

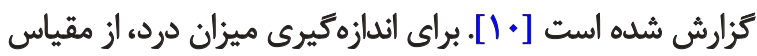

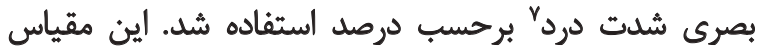

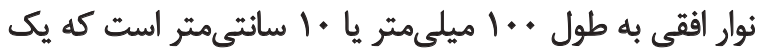

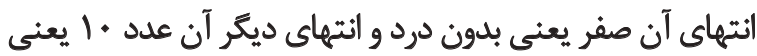

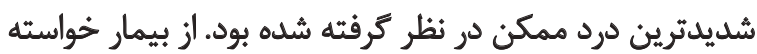

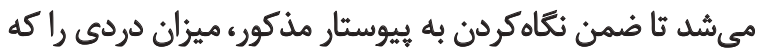

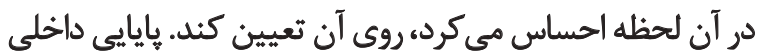

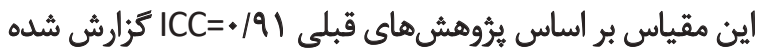

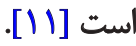

\section{اندازهتيرى ميزان فعاليت بدنى}

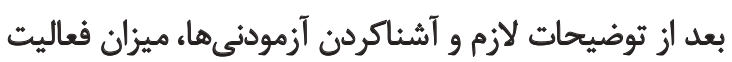

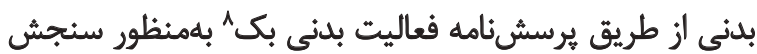

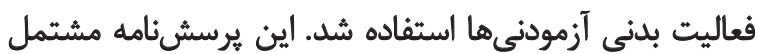

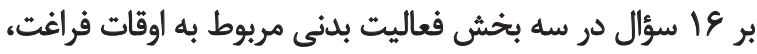

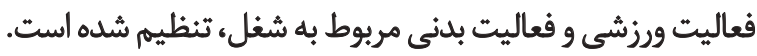

6. Keele STarT Back

7. Visual Analogue Scale (VAS)

8. Beck Physical Activity Questionnaire
قسمت ميانى بدن ممكن است موجب افزايش قوس كمرى شود،

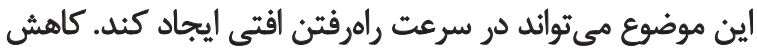

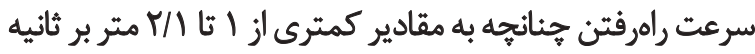

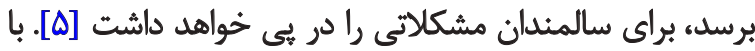

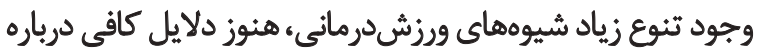
برترى يك روش بر ديكرى وجود ندارد.

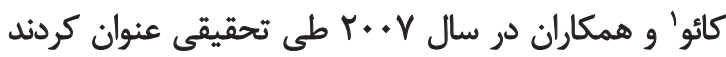

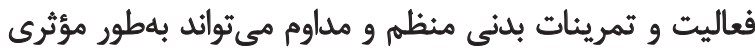

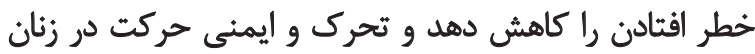

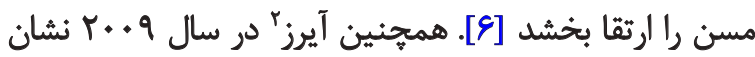

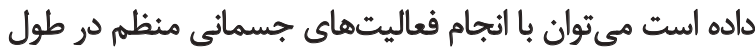

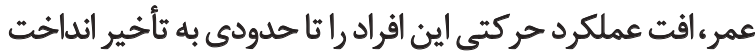

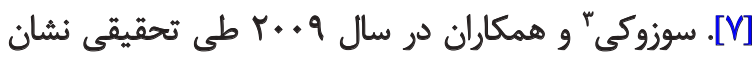

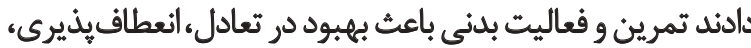

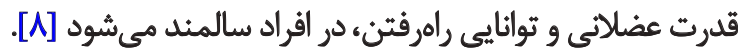
با توجه به تحقيقات صورت ترفته، مشخص است درباره ورزش

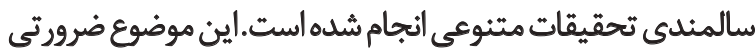

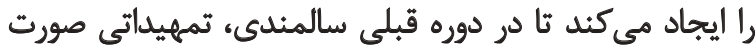

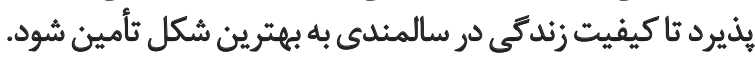

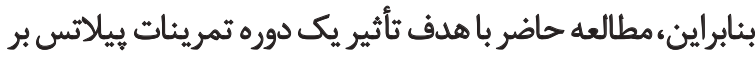

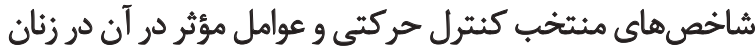
سالمند غير فعال انجام شد. مند.

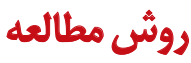

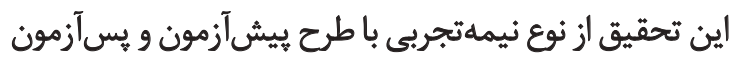

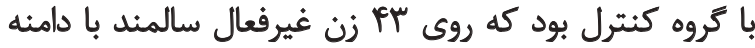

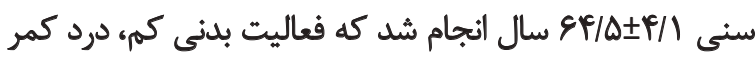

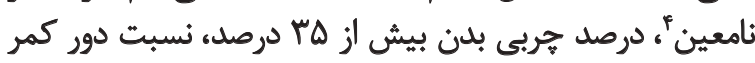

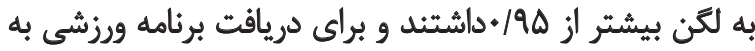

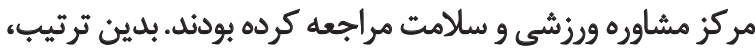

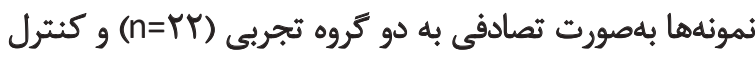

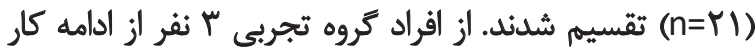
منصرف شدند.

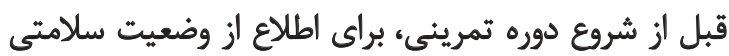

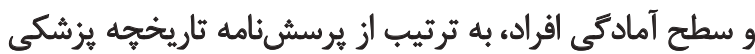

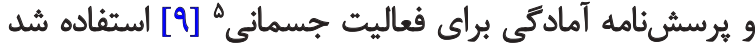

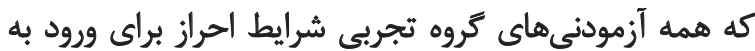

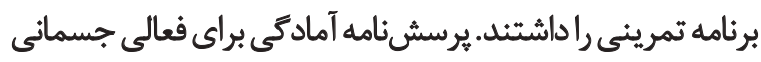

\section{Cao}

2. Irez

3. Suzuki

4. Non-Specific Low Back Pain

5. Physical Activity Readiness Questionnaire (PARQ) 


\section{برنامل تمرينى}

براي كروه تجربى كه تحت تمرينات بيلاتس قرار كرفتند،

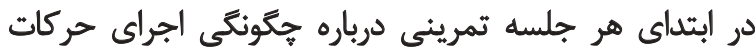

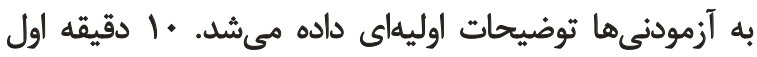

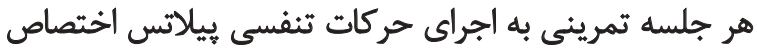

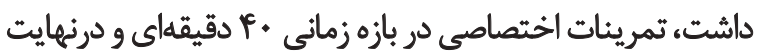

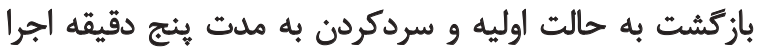

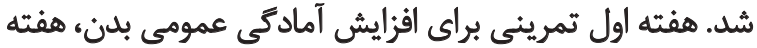

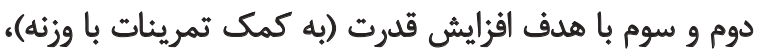

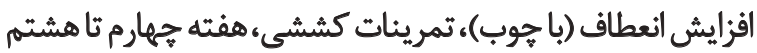

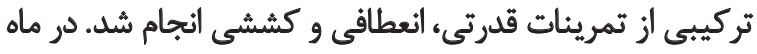

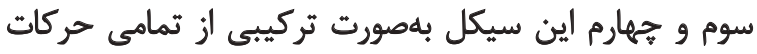

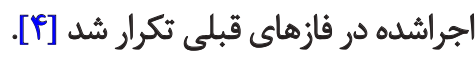

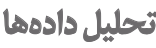

در مطالعه حاضر از آزمون آمارى شاييرو- ويلك براى ارزيابى إزيى

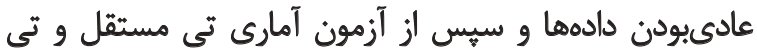

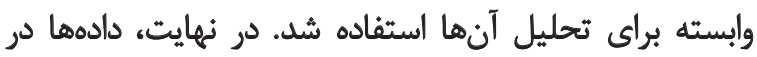

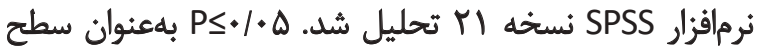
معنادارى در نظر ترفته شد.

\section{يافتّهها}

بنتايج آزمون t نشان داد حجم عضله آزمودني هايي كه فعاليت

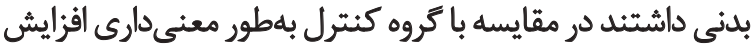

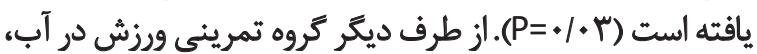

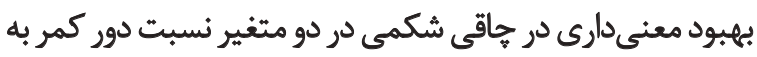

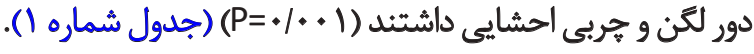
همانطور كه در تصوير شماره ( مشاهده مىشود، الف)

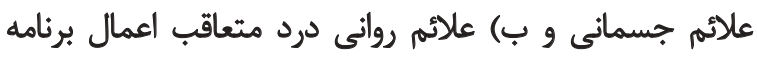

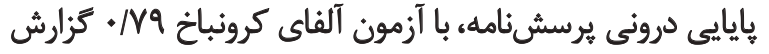

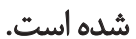

$$
\text { اندازهتيرى تر كيب بدنى }
$$

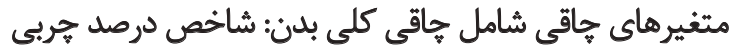

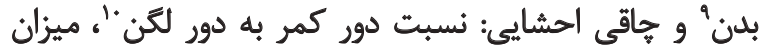

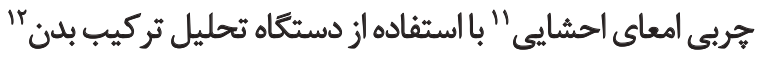

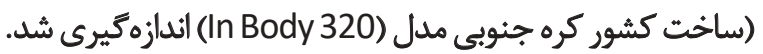

$$
\text { أندازهيّيرى + (متر سرعت راه رفتن }
$$

براى اندازهيرى سرعت آزمودنىهاء از آنها خواسته شد كه

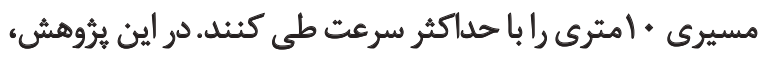

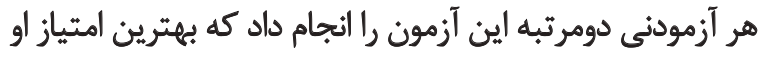

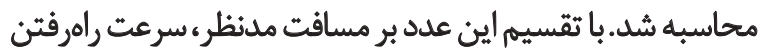
فرد برحسب متر بر ثانيه به دست آمد.

$$
\text { أزمون تعادل (زمان برخاستن و وقثتي") }
$$

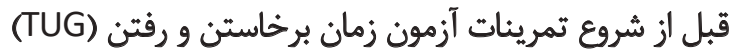

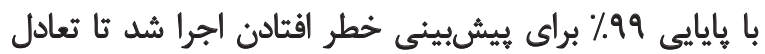

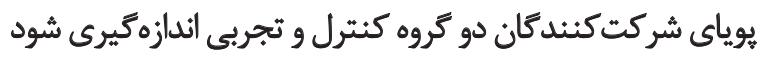

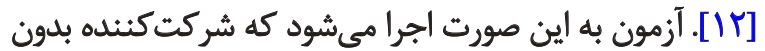

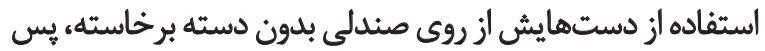

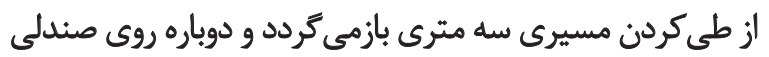

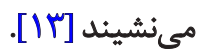

9. Percentage of Body Fat (PBF)

10. Waist Hip Ratio (WHR)

11. Visceral fat

12. Body composition analyzer

13. Time up and go

جدول ا. ويثُكىهاى عمومى و آنترويومتريكى آزمودنىها در دو كروه تجبربى و كنترل

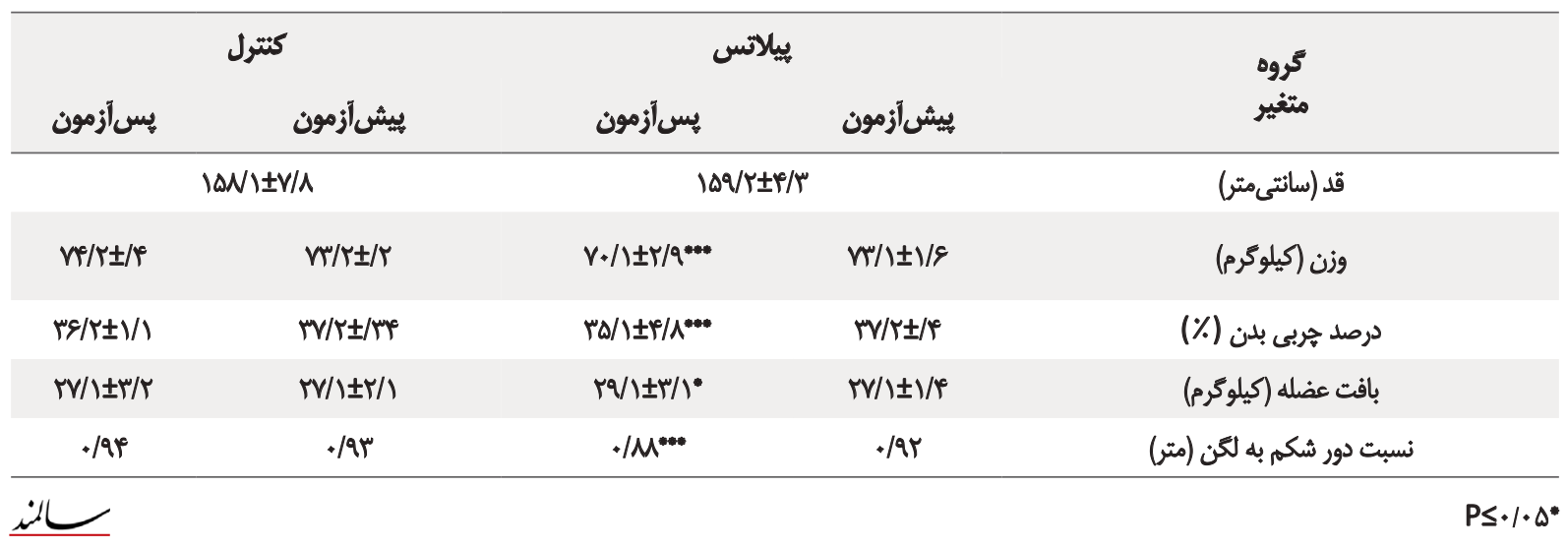

$P \leq+1 . .1 * * *$ 
جدول r. آزمون t وابسته جهت بررسى ميزان تعادل يويا قبل و بعد از مداخله

\begin{tabular}{|c|c|c|c|c|}
\hline معنادارى سطع & مقدار & & & كو \\
\hline $.1 .+1 *$ & EIDQ & $V / \Delta P \pm \cdot / M$ & 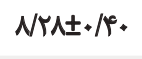 & تجربي \\
\hline.$|8|$ &.$(\Delta) 1$ & v/ut./rq & $N \cdot \Delta \pm \cdot|f|$ & كتترل \\
\hline
\end{tabular}

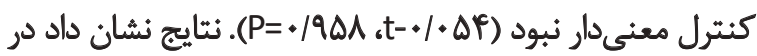

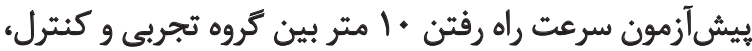

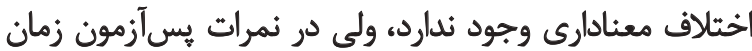

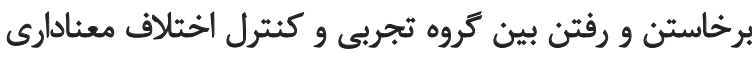

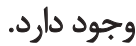

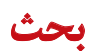

هدف از مطالعه حاضر، بررسى تأثير تمريئات بيلاتس بر بر

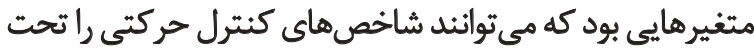

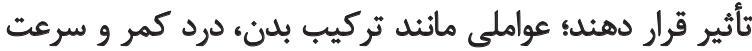

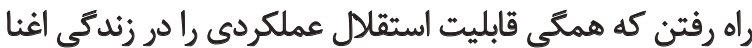
مى كنيند. نتايج بهدست آمده نشان

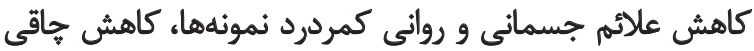

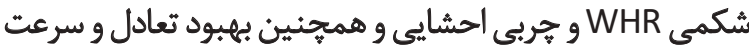
راه رفتن مي شود.

جنانكه در مبانى نظرى تحقيق عنوان شد ارتباط مستقيمى

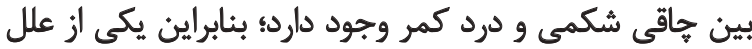

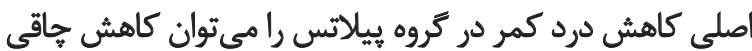

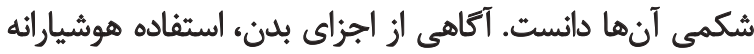

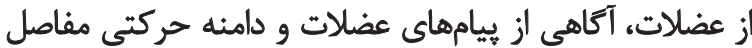

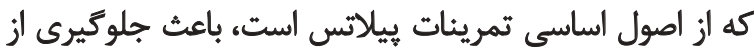

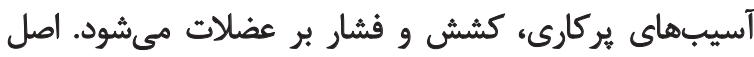

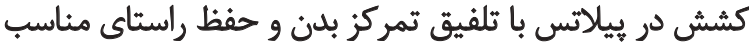
بدن كمك مي كند تا عضلات در دامنه حركتي كئ كامل كه بـ براي

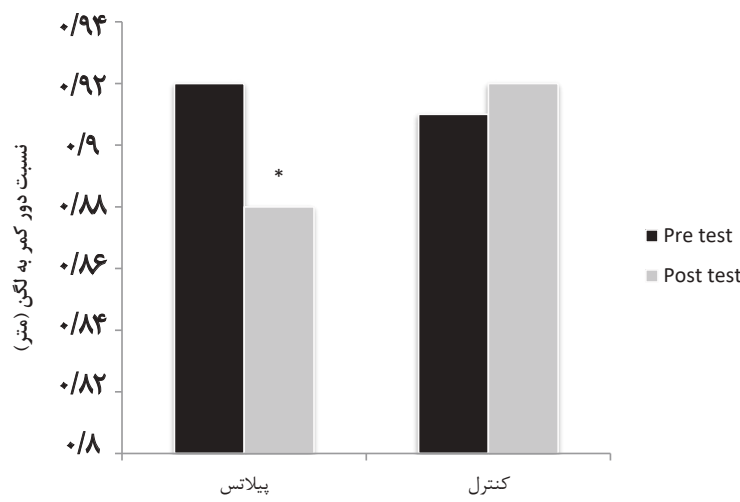

تصوير r. تأثير بيلاتس بر نسبت دور كمر به لكن $\quad$ ل

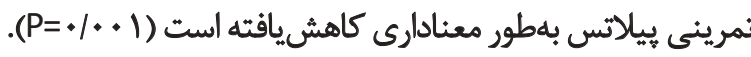

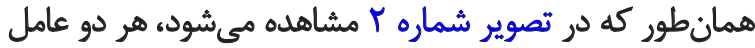

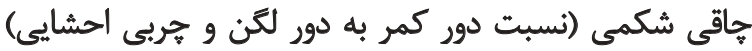

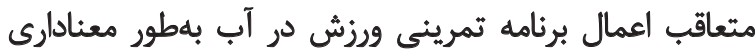

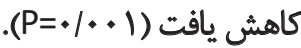
نتايج آزمون t وابسته در جدول شماره Y آمده است. تعادل

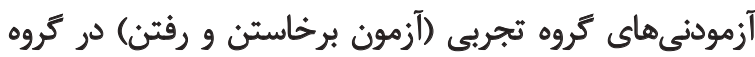

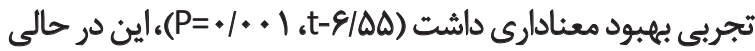

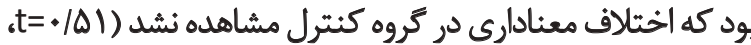

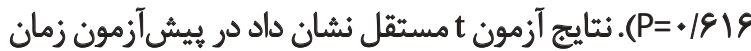

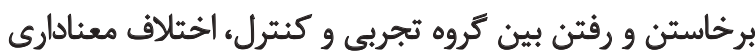

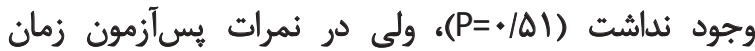

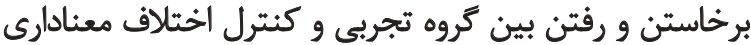

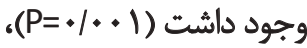

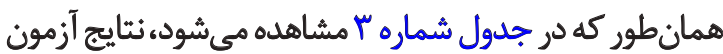

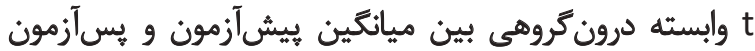

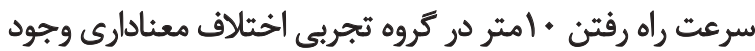

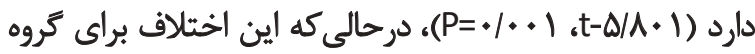
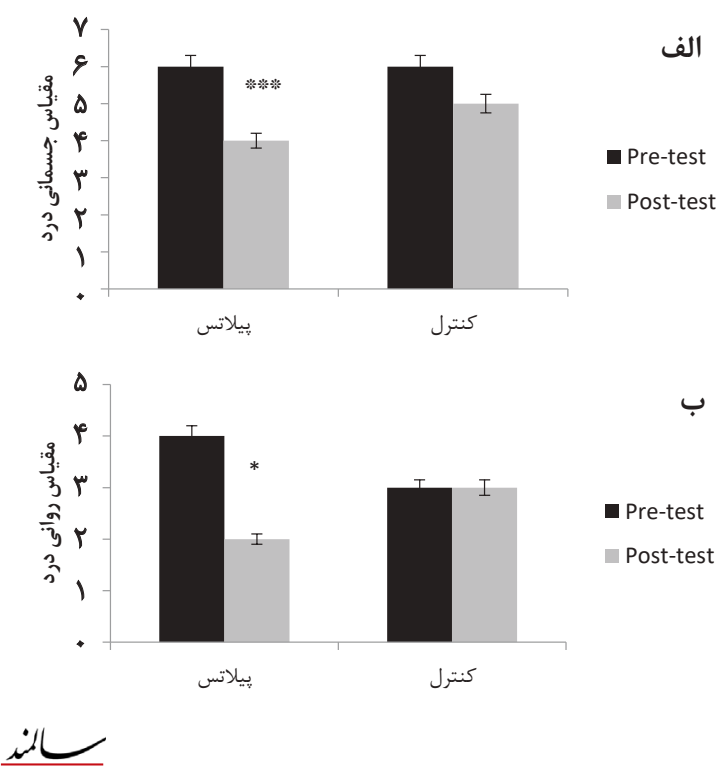

تصوير ا. الرزيابى درد كمر در كروههاى تحقيق

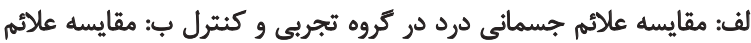
روانى درد در كروه تجربى و كنترل 
جدول r. آزمون t وابسته جهت بررسى ميزان سرعت آزمودنىها قبل و بعد از مداخله

\begin{tabular}{|c|c|c|c|c|}
\hline معنادارى & مقدار & & & مروه \\
\hline $.1 . .1^{*}$ & $\Delta / \Delta \cdot 1$ & $1 / A \Delta \pm \cdot / \cdot f$ & $1 / 9 \cdot \pm \cdot / \cdot r$ & تجربى \\
\hline.$/ 9 \Delta \mathrm{A}$ & $.1 . \Delta f^{e}$ & $1 / 9 r \pm . / . r$ & $1 / 91 \pm \cdot 1 \cdot p^{e}$ & كثترل \\
\hline
\end{tabular}

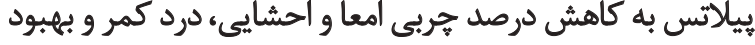

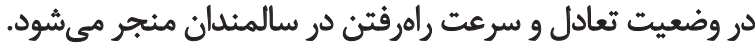

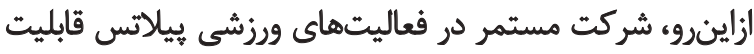

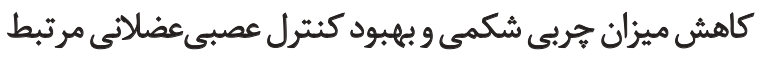

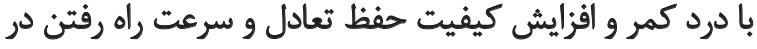

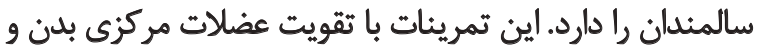

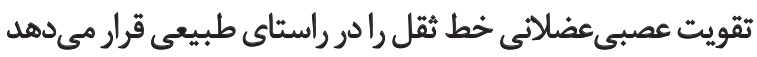

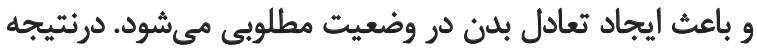

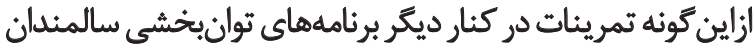
مي توان استفاده كرد. تمرينات درن

مالامظاث اخاقلى

\section{ييروى از اصول اخالاق يُوهش}

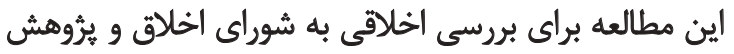

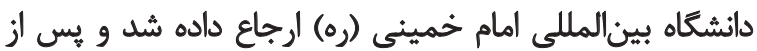

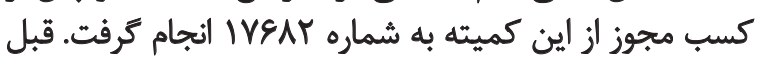

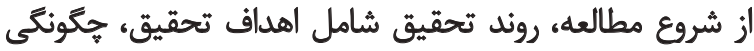

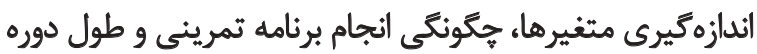
تحقيق براى آزمونىى ها توضيح داده شد. تمامى شركت كت كنيندكان

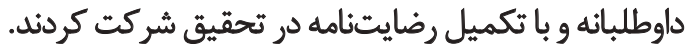

$$
\text { كامي مالي }
$$

اين مقاله حامى مالى ندارد.

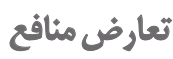

بنا بر اظهار نظر نويسندكان اين مقاله تعارض منافع ندارد.

$$
\text { تشكر وقدردانى }
$$

بدينوسيله از زحمات تمامي افرادى كه در اين مطالعه شركت

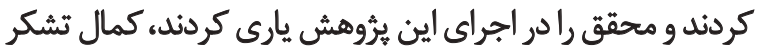
و قدرداني هي شيود.
تعادل عضلاتي لازم است، كشيده شوند و همزمان عمل انقباض

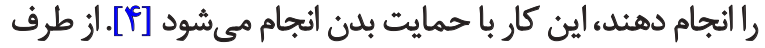

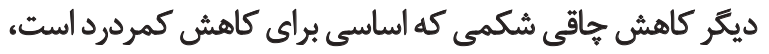

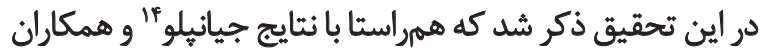

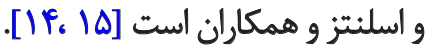

همجينين بهبود در وضعيت حفظ تعادل و سرعت راهرفتن بائ

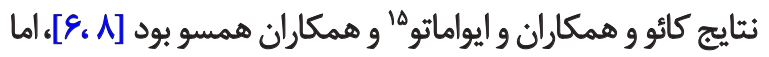

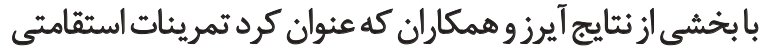

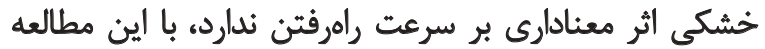

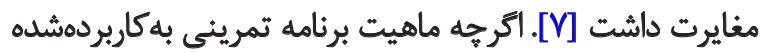

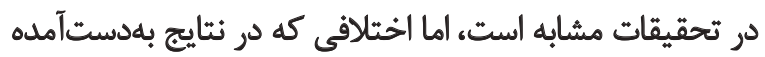

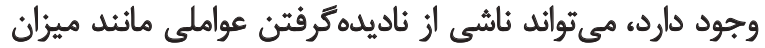

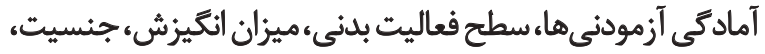

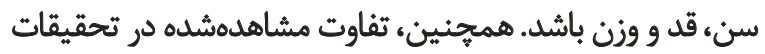

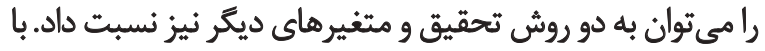

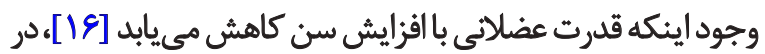

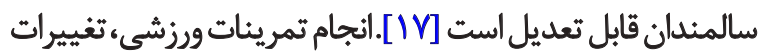

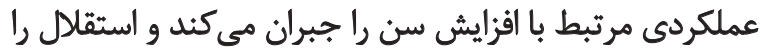

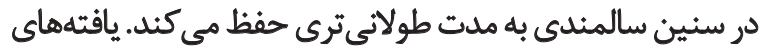

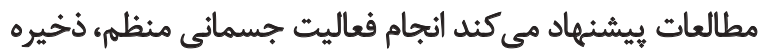

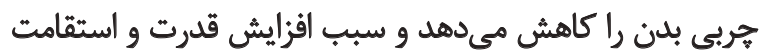

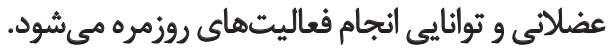

آتروفي عضلاني نيز كه با افزايش سن همراه است، از طريق

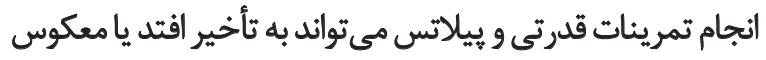

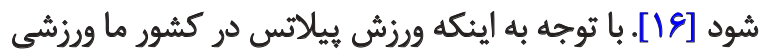

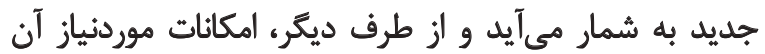
بسيار سهل الوصول است و در هر مكانى قابليت اجرا دارد، توصيه مي شود براى افزايش آمادكى عملكردى ازيت إجمله سرعت، تعادل

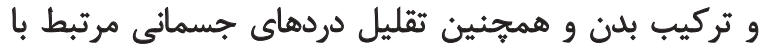
كمردرد داستفاده شود.

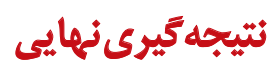
با توجه به نتايج يرُوهش حاضر، مىتوان كفت كه تمرينات 


\section{References}

[1] Nachemson A, Waddell G, Norlund A. Epidemiology of neck and low back pain. In: Nachemson A, Jonsson E, editors. Neck and Back Pain: The Scientific Evidence of Causes, Diagnos is and Treatment. Philadelphia, Pennsylvania: Lippincott Williams \& Wilkins; 2000 .

[2] Ebenbichler GR, Oddsson LI, Kollmitzer J, Erim Z. Sensorymotor control of the lower back: Implications for rehabilitation. Medicine \& Science in Sports \& Exercise. 2001; 33(11):1889-98. [DOI:10.1097/00005768-200111000-00014]

[3] Wright VJ, Perricelli BC. Age-related rates of decline in performance among elite senior athletes. The American Journal of Sports Medicine. 2008; 36(3):443-50. [DOI:10.1177/0363546507309673] [PMID]

[4] Critchley DJ, Pierson Z, Battersby G. Effect of pilates mat exercises and conventional exercise programmes on transversus abdominis and obliquus internus abdominis activity: Pilot randomised trial. Manual Therapy. 2011; 16(2):183-9. [DOI:10.1016/j. math.2010.10.007] [PMID]

[5] Hoxie RE, Rubenstein LZ. Are older pedestrians allowed enough time to cross intersections safely? Journal of the American Geriatrics Society. 1994; 42(3):241-4. [DOI:10.1111/j.1532-5415.1994. tb01745.x]

[6] Cao ZB, Maeda A, Shima N, Kurata H, Nishizono H. The effect of a 12-week combined exercise intervention program on physical performance and gait kinematics in community-dwelling elderly women. Journal of Physiological Anthropology. 2007; 26(3):32532. [DOI:10.2114/jpa2.26.325]

[7] Irez B. Pilates exercise positively affects balance, reaction time, muscle strength, number of falls and psychological parameters in 65+ years old women [PhD dissertation]. Ankara: Middle East Technical University; 2009.

[8] Iwamoto J, Suzuki H, Tanaka K, Kumakubo T, Hirabayashi H, Miyazaki Y, et al. Preventative effect of exercise against falls in the elderly: A randomized controlled trial. Osteoporosis International. 2009; 20(7):1233-40. [DOI:10.1007/s00198-008-0794-9] [PMID]

[9] Thomas S, Reading J, Shephard RJ. Revision of the Physical Activity Readiness Questionnaire (PAR-Q). Canadian Journal of Sport Sciences. 1992; 17(4):338-45. [PMID]

[10] Hill JC, Dunn KM, Main CJ, Hay EM. Subgrouping low back pain: a comparison of the STarT Back Tool with the Örebro Musculoskeletal Pain Screening Questionnaire. European Journal of Pain. 2010; 14(1):83-9. [DOI:10.1016/j.ejpain.2009.01.003] [PMID] [PMCID]

[11] Karimi A. A prospective study of the outcome of treatment of chronic low back pain patients with consistent and inconsistent clinical signs as defined by three screening tests [MSc. thesis]. Norwich: University of East Anglia; 2004.

[12] Lopopolo RB, Greco M, Sullivan D, Craik RL, Mangione KK. Effect of therapeutic exercise on gait speed in community-dwelling elderly people: A meta-analysis. Physical Therapy. 2006; 86(4):520-40. [PMID]

[13] Buchner DM, Cress ME, De Lateur BJ, Esselman PC, Margherita AJ, Price R, et al. The effect of strength and endurance training on gait, balance, fall risk, and health services use in community-living older adults. The Journals of Gerontology Series A: Biological Sciences and Medical Sciences. 1997; 52(4):M218-M24. [DOI:10.1093/gerona/52A.4.M218] [PMID]
[14] Slentz CA, Duscha BD, Johnson JL, Ketchum K, Aiken LB, Samsa GP, et al. Effects of the amount of exercise on body weight, body composition, and measures of central obesity: STRRIDE a randomized controlled study. Archives of internal medicine. 2004; 164(1):31-9. [DOI:10.1001/archinte.164.1.31] [PMID]

[15] Giannopoulou I, Ploutz-Snyder L, Carhart R, Weinstock R, Fernhall B, Goulopoulou S, et al. Exercise is required for visceral fat loss in postmenopausal women with type 2 diabetes. The Journal of Clinical Endocrinology \& Metabolism. 2005; 90(3):1511-8. [DOI:10.1210/jc.2004-1782] [PMID]

[16] Md JFB, Kiely DK, Herman S, Leveille SG, Mizer K, Frontera WR, et al. The relationship between leg power and physical performance in mobility-limited older people. Journal of the American Geriatrics Society. 2002; 50(3):461-7. [DOI:10.1046/j.1532 5415.2002.50111.x]

[17] Rogers ME, Rogers NL, Takeshima N, Islam MM. Methods to assess and improve the physical parameters associated with fall risk in older adults. Preventive Medicine. 2003; 36(3):255-64. [DOI:10.1016/S0091-7435(02)00028-2] 